\title{
Horizontal Vibrations of Embedded Foundation in Multi-Layered Poroelastic Soils
}

\author{
Suraparb Keawsawasvong, Teerapong Senjuntichai* \\ Applied Mechanics and Structures Research Unit, Department of Civil Engineering, Chulalongkorn University, THAILAND \\ "Corresponding author: teerapong.s@chula.ac.th
}

SUBMITTED 25 April 2019 REVISED 26 April 2019 ACCEPTED 29 April 2019

\begin{abstract}
In this paper, the dynamic response of rigid foundations of arbitrary shape embedded in multi-layered poroelastic soils subjected to time-harmonic horizontal loading is presented. The soil-structure interaction problem is investigated by employing a discretization technique and flexibility equations based on the influence functions obtained from an exact stiffness matrix scheme. The present solution scheme is verified with relevant existing solutions of rigid foundations on homogeneous elastic and poroelastic media. A selected set of numerical results are illustrated to portray the influence of various parameters, namely, frequency of excitation, poroelastic material parameters, foundation shapes, embedded depth, and the supporting soil systems, on nondimensional horizontal compliances of rigid foundations.
\end{abstract}

KEYWORDS Foundations; horizontal vibrations; Multi-layered soils; Poroelasticity; Soil-structure interaction; Time-harmonic

(c) The Author(s) 2018. This article is distributed under a Creative Commons Attribution-ShareAlike 4.0 International license.

\section{INTRODUCTION}

An important class of problems encountered in geotechnical engineering, earthquake engineering, and offshore engineering is concerned with soilstructure interaction analysis of a foundation under horizontal dynamic loading from an earthquake, wind, ocean wave, and machine vibration. In the past, several researchers employed a variety of techniques to study horizontal vibrations of rigid foundations. For example, (Luco and Westmann (1972) considered the case of a rigid strip bonded to a homogenous elastic medium and subjected to time-harmonic loading. Coupled rocking and sliding responses of a rectangular plate were investigated by (Urlich and Kuhlemeyer (1973), who employed a finite element method with an energy absorbing boundary. Wong and Luco (1976) presented a time-harmonic horizontal motion of a rectangular foundation resting on an elastic half-space. In addition, horizontal vibrations of rectangular foundations bonded to an elastic medium were also studied by Wong and Luco (1978). Generally, geomaterials are two-phased materials consisting of a solid skeleton with voids filled with water. Such materials are commonly known as poroelastic materials, and they are considered to be a more suitable representation of soils and rocks than ideal elastic materials.
The theory of elastic wave propagations in a poroelastic medium was presented by Biot (1956) by adding the inertia terms to his quasi-static theory (Biot, 1941). For horizontal vibrations of foundations in poroelastic soils, Kassir and Jimin, 1988) (1988) presented the case of rigid strip foundation on a poroelastic half-plane. Horizontal vibrations of a rigid circular foundation under time-harmonic loading resting on a poroelastic half-space were presented by Jin and Liu (2000). In addition, He and Wang (2013) and He and Zhou (2015) studied horizontal vibrations of an underwater rigid disk on a poroelastic half-space. All existing works mentioned above are concerned with horizontal vibrations of foundations on homogeneous poroelastic soils. However, natural soil profiles normally consist of a number of soil layers with different thicknesses and material properties. A review of literature indicates that dynamic interaction between a rigid foundation subjected to horizontal loading and multi-layered poroelastic soils has never been reported in the past.

In this paper, the dynamic response of a rigid foundation, subjected to time-harmonic horizontal loading and embedded in multi-layered poroelastic soils (see Figure 1), is presented. The foundation is assumed to be massless, and fully permeable. In addition, it is perfectly bonded to the supporting soils. The soil medium under consideration consists of a number of poroelastic layers of different 
thicknesses and material properties, and each layer is governed by Biot's theory of poroelastodynamics. A computer program based on the proposed solution scheme has been developed, and its accuracy has been verified by comparing with existing studies on horizontal vibrations of rigid foundations. Selected numerical results are presented to demonstrate the influence of foundation shape, embedded depth, poroelastic material properties, and the supporting soil systems on horizontal compliances of rigid foundations.

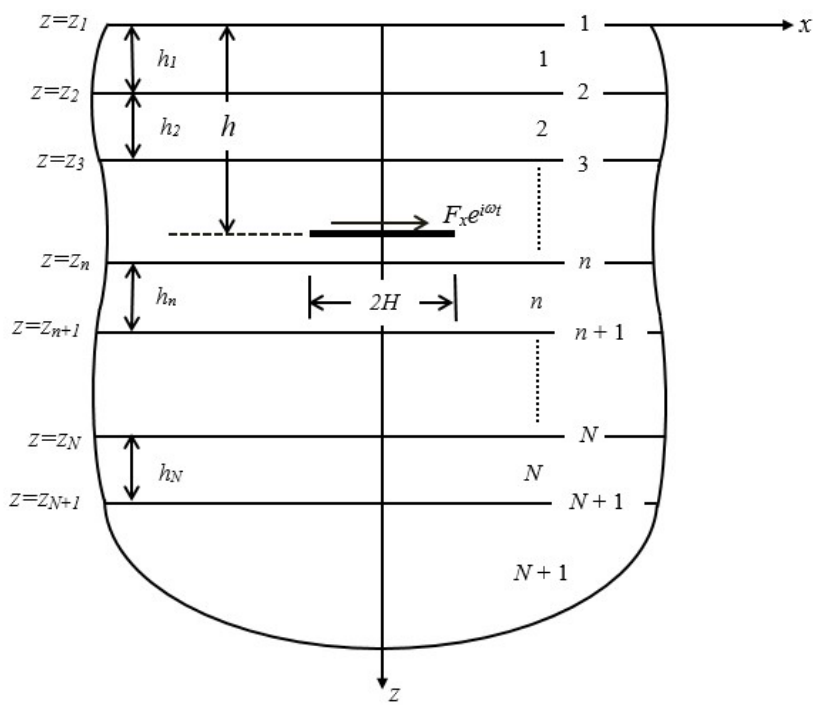

Figure 1. Schematic of rigid foundation in multi-layered poroelastic soils under horizontal loading.

\section{BASIC EQUATIONS}

Consider a multi-layered poroelastic half-space with a Cartesian coordinate system $(i=x, y, z)$ defined as shown in Figure 1. Let $u_{i}(x, y, z, t)$ and $w_{i}(x, y, z, t)$ denote the average displacement of the solid matrix and the fluid displacement relative to the solid matrix in the $i$-direction $(i=x, y, z)$ respectively. The constitutive relation of a poroelastic material can be expressed as in Equation 1 (Biot, 1941),

$\sigma_{i j}=2 \mu \varepsilon_{i j}+\lambda \delta_{i j} \varepsilon_{k k}-\alpha \delta_{i j} p i, j=x, y, z$

$p=-M\left(\alpha \varepsilon_{k k}+w_{i, i}\right)$

where $\sigma_{i j}$ is the total stress component of the bulk material; $\varepsilon_{i j}$ is the strain component; $\mu$ and $\lambda$ denote Lame' constants of the bulk material; $\delta_{i j}$ is the Kronecker delta; and $p$ denotes the excess pore fluid pressure. In addition, $\alpha$ and $M$ are Biot's parameters accounting for compressibility of the two-phased material.
In this study, the motion is assumed to be time-harmonic with the factor of $e^{i \omega t}$, where $\omega$ is the frequency of excitation. The equations of motions for the two-phase material (Biot, 1962), in the absence of body forces and a fluid source, can then be expressed in this case as,

$$
\begin{array}{r}
\mu u_{i, j j}+\left(\lambda+\alpha^{2} M+\mu\right) u_{j, j i}+\alpha M w_{j, j i} \\
=-\omega^{2} \rho u_{i}-\omega^{2} \rho_{f} w_{i} \\
\alpha M u_{j, j i}+M w_{j, j i}=-\omega^{2}\left(\rho_{f} u_{i}+m w_{i}\right)+i \omega b w_{i}
\end{array}
$$

where $\rho$ and $\rho_{f}$ are the mass densities of the bulk material and the pore fluid, respectively; and $m$ is a density-like parameter that depends on $\rho_{f}$ and the geometry of the pores. In addition, $b$ is a parameter accounting for the internal friction due to the relative motion between the solid matrix and the pore fluid.

The general solutions to the equations of motions, Equation 2, can be determined by applying Helmholtz representation together with the double Fourier integral transform with respect to the horizontal coordinates given in Equation 3 (Sneddon, 1951),

$\bar{f}\left(k_{x}, k_{y}, z\right)=\int_{-\infty}^{\infty} \int_{-\infty}^{\infty} f(x, y, z) e^{-i k_{x} x-i k_{y} y} d x d y$

where $k_{x}$ and $k_{y}$ are the wave numbers associated with the $x$ and $y$ coordinates respectively. The inverse relationship is given by,

$f(x, y, z)=\frac{1}{(2 \pi)^{2}} \int_{-\infty}^{\infty} \int_{-\infty}^{\infty} \bar{f}\left(k_{x}, k_{y}, z\right) e^{i k_{x} x+i k_{y} y} d k_{x} d k_{y}$

The general solutions in the Fourier transform domain can be found elsewhere (Lu and Jeng, 2007).

In the present study, the analysis of the dynamic interaction problem shown in Figure 1 requires a set of influence functions of a multi-layered half-space subjected to tangential traction of unit intensity applied in the $x$ direction over a square area at the depth $h$ below the free surface of the half-space. An exact stiffness scheme proposed by Senjuntichai, Keawsawasvong and Plangmal (2018) is employed to obtain the required influence functions. Consider a multi-layered poroelastic halfspace consisting of $N$ poroelastic layers $(1,2, . ., N)$ and an underlying poroelastic half-space as shown in Figure 1. The stiffness matrices for each layer and the half-space are obtained explicitly from the relationship between the generalized displacements and stresses obtained from the analytical general solutions. By enforcing the continuity of displacements and tractions at each layer interface, the global stiffness matrix can then be constituted. The numerical solution of the global stiffness equation for a multi-layered poroelastic half-space under 
horizontal traction applied uniformly over a square area yields the required influence functions for the proposed solution scheme. More details of the exact stiffness matrix scheme together with the explicit expressions of all relevant matrices are shown in (Senjuntichai, Keawsawasvong and Plangmal (2018).

\section{FORMULATION OF INTERACTION PROBLEM}

Consider a rigid foundation of arbitrary shape with a width of $2 \mathrm{H}$, subjected to a time-harmonic horizontal load in the $x$-direction of amplitude $F_{\mathrm{x}}$, embedded at a depth $h$ below the surface of multi-layered poroelastic soils as shown in Figure 1. The foundation is assumed to be massless, fully permeable, and undergoing a time-harmonic horizontal displacement of amplitude $\Delta_{x}$. Let $T_{x}$ denotes the tangential traction generated at the contact surface under the rigid foundation. The discretization technique proposed by Wong and Luco (1976) is employed to solve for the unknown $T_{x}$. By using this technique, the rectangular foundation of size $2 H \times 2 L$ as shown in Figure 2(a) is divided into a number of $N_{x}$ $\times N_{y}$ square elements where $N_{x}$ and $N_{y}$ denote the number of square elements in the $x$ - and $y$-direction respectively. It is assumed that $T_{x}$ is constant within each discretized element. Note that the nodal point of each element is located at its center. The following relationship can then be established on the discretized contact surface under the rigid rectangular foundation:

$\Delta_{x}=\sum_{n=1}^{N_{y}} \sum_{m=1}^{N_{x}}\left[G_{x x}\left(x_{k}, y_{l} ; x_{m}, y_{n}\right) T_{x}\left(x_{m}, y_{n}\right)\right]$

where $G_{x x}\left(x_{k}, y_{l} ; x_{m}, y_{n}\right)$ denotes the influence function, which is the horizontal displacement at a point with coordinate $\left(x_{k}, y_{l}, h\right)$ due to a uniform horizontal load of unit intensity applied at a discretized element with the nodal point $\left(x_{m}, y_{n}, h\right)$. The influence function can be obtained from the exact stiffness matrix scheme Senjuntichai, Keawsawasvong and Plangmal (2018a). The solution to Equation 4 yields the unknown $T_{x}$ at each discretized element and the horizontal load $F_{\mathrm{x}}$ can then be computed from the following equation:

$F_{x}=\int_{-L}^{L} \int_{-H}^{H}\left[T_{x}(x, y)\right] d x d y$

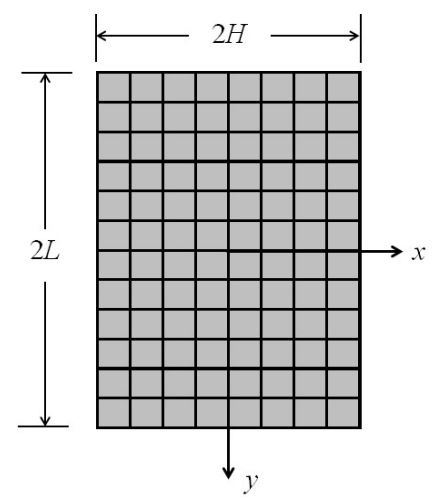

(a)
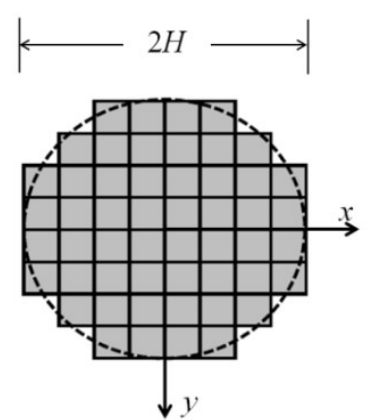

(b)

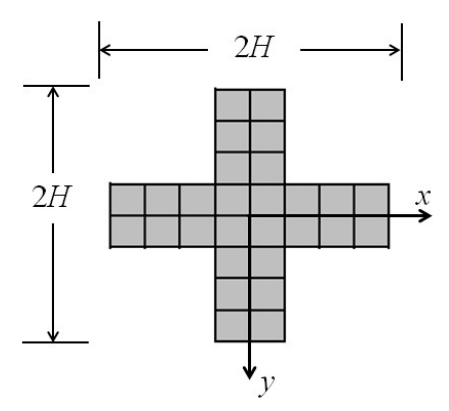

(c)

Figure 2. Discretization of contact areas under various rigid foundations considered in the numerical study: (a) rectangular; (b) circular; and (c) cruciform.

Finally, the horizontal vibration of rigid foundation can be characterized by the following nondimensional horizontal compliance,

$C_{H}=\frac{\mu^{(1)} H \Delta_{x}}{F_{x}}$

where $\mu^{(1)}$ is the shear modulus of the first layer of the multi-layered soils. 

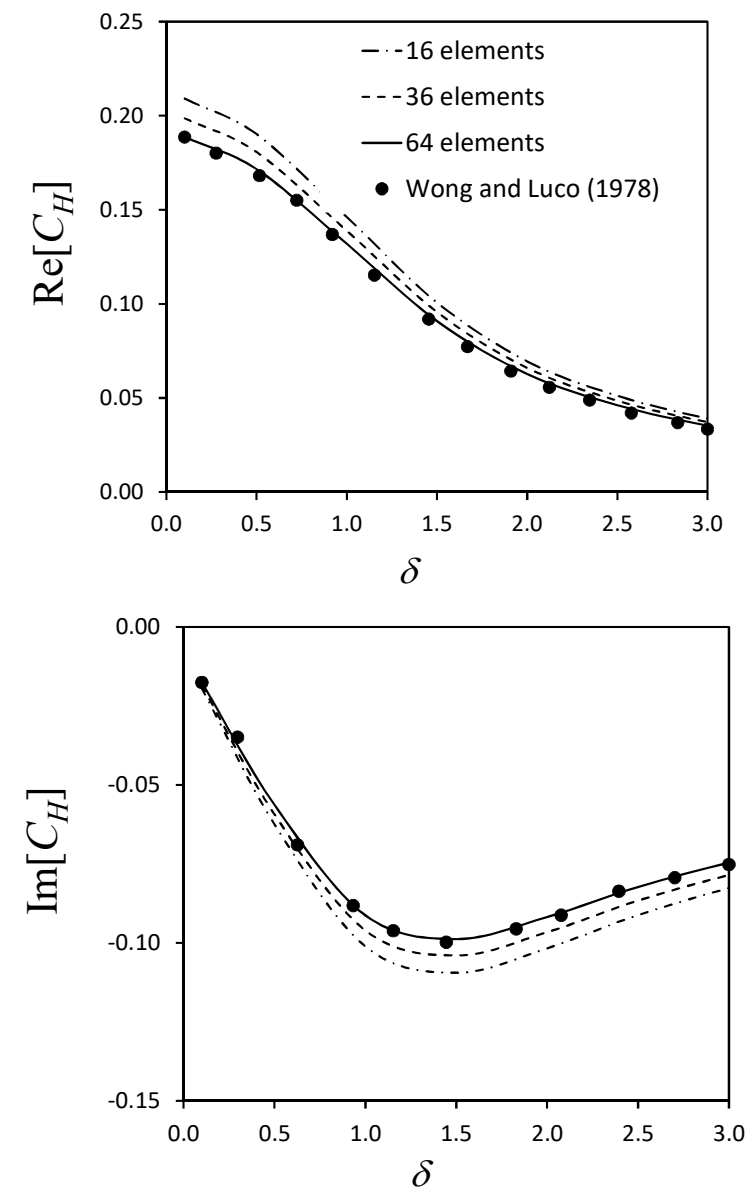

Figure 3. Comparison of non-dimensional horizontal compliances of square foundation on elastic half-space.

\section{NUMERICAL RESULTS AND DISCUSSION}

This section presents numerical results for dynamic compliances of embedded rigid foundations in multilayered poroelastic soils subjected to time-harmonic horizontal loading as shown in Figure 1. To obtain the required influence functions employed in the formulation of the interaction problem outlined in the foregoing section, the inversion of the doubleFourier transform of the horizontal displacement, given by Equation (3b), must be performed. The globally adaptive numerical quadrature scheme (Piessens et al., 2012) is employed to perform the numerical evaluation of the semi-infinite integral in each direction. This scheme subdivides the interval of the integrand and uses a 21-point Gauss-Kronrod rule to estimate the integral over each interval. The subdivision continues until the specified tolerance of $10^{-3}$ from the approximation is attained. In the numerical study, a non-dimensional frequency $\delta=$ $\omega H \sqrt{\rho^{(1)} / \mu^{(1)}}$ is employed where $\rho^{(1)}$ denotes the mass density of the first layer.
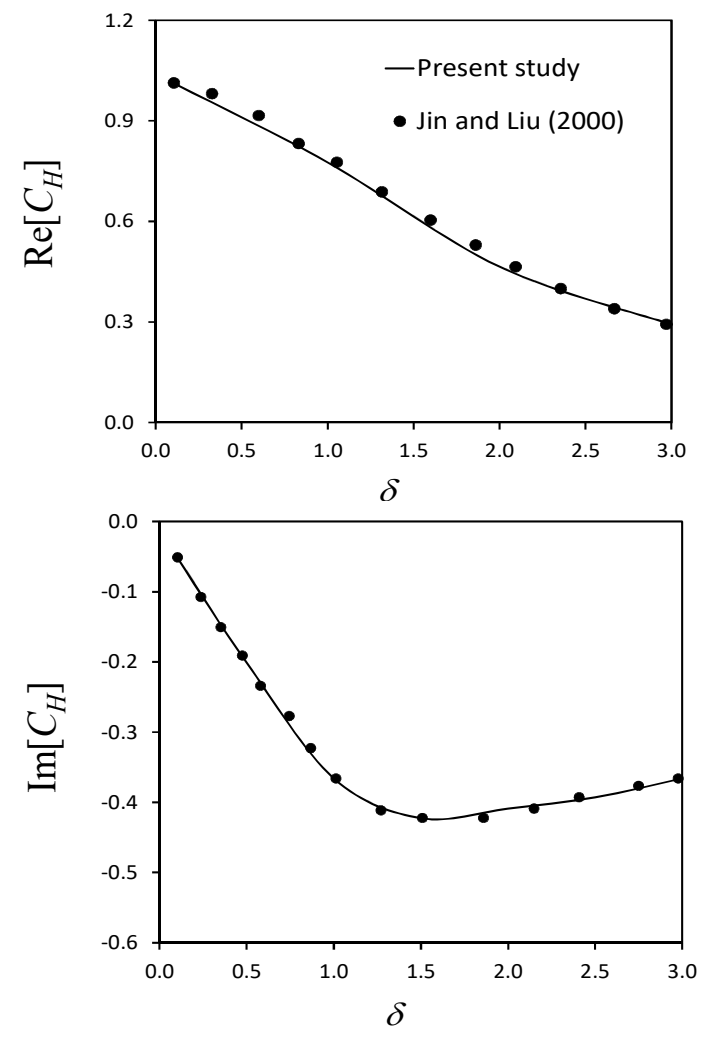

Figure 4. Comparison of non-dimensional horizontal compliances of circular foundation on poroelastic halfspace.

In order to verify the convergence and accuracy of the present solution scheme, the existing solution of a rigid square foundation given by Wong and Luco (1978) is employed. Figure 3 shows a comparison of nondimensional horizontal compliance of a rigid square foundation of size $2 H \times 2 H$ [see Figure 2(a) with $L=H$ ] resting on a homogenous elastic half-space with Poisson's ratio of 0.33 between the present solution and the solution by Wong and Luco (1978). To show the convergence of the present study, the contact area under the square foundation is divided into different numbers of discretized elements, i.e. $N_{x} \times N_{y}$ $=4 \times 4$ or 16 elements, $6 \times 6$ or 36 elements, and $8 \times 8$ or 64 elements. It is found that the solutions from the present study agree well with Wong and Luco (1978) when $N_{x} \times N_{y} \geqslant 64$. Non-dimensional horizontal compliances of a rigid circular foundation of radius $H$ resting on a homogenous poroelastic half-space were presented by Jin and Liu (2000). The material properties of the half-space are given as follows: $\lambda / \mu=$ $1.5 ; M / \mu=10 ; \rho_{f} / \rho=0.53 ; \mathrm{m} / \rho=1.1 ; \alpha=0.97$ and $b H /(\rho \mu)^{0.5}$ $=0.1$. The number of square elements used to discretize the contact area under the circular foundation in the present solution is 52 , which are obtained by removing some elements near the corners of the discretized square contact area of 64 elements, as shown in Figure 2(b). In Figure 4, it is obvious that 
a very good agreement between the two solutions is obtained throughout the frequency range under consideration.
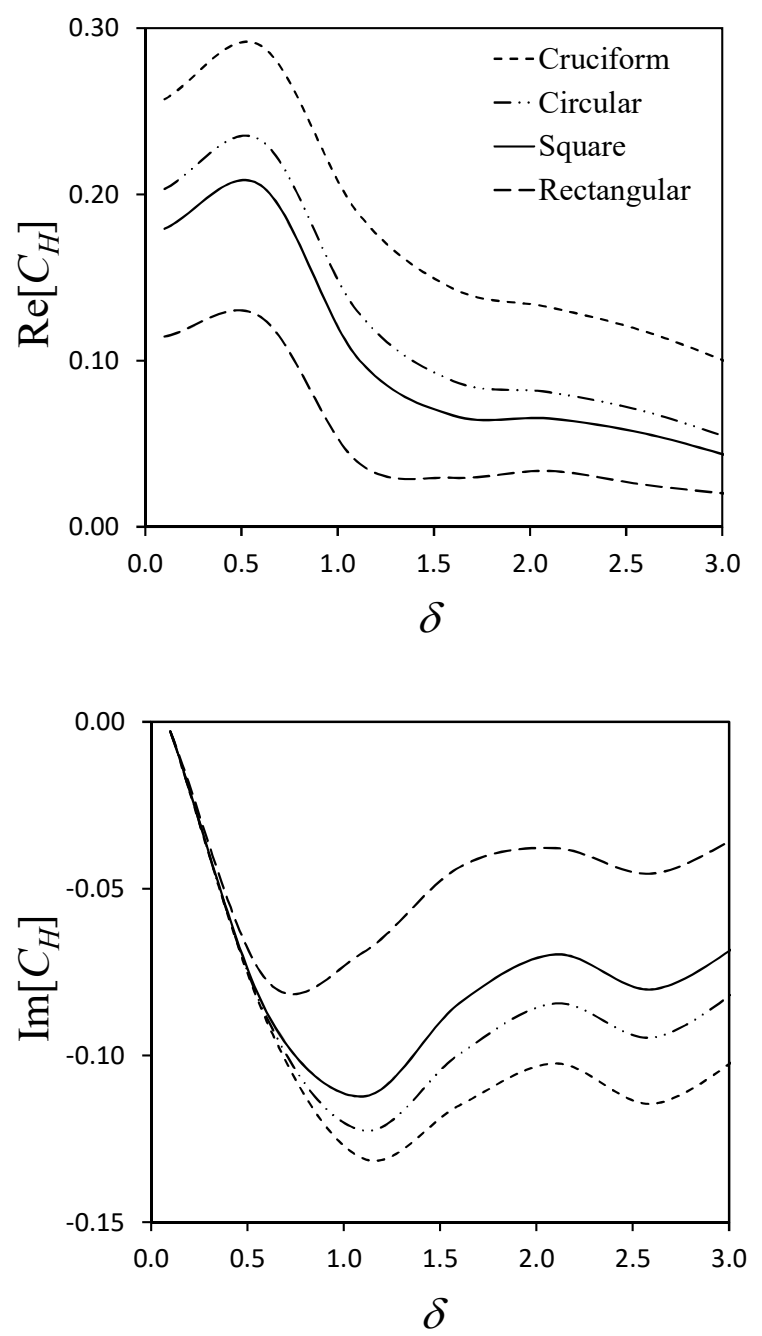

Figure 5. Non-dimensional horizontal compliances of rigid foundations of various shapes.

Non-dimensional horizontal compliances of rigid foundations of various shapes in multi-layered poroelastic soils over the frequency range $0.1<\delta<3$ are considered next. The multi-layered poroelastic soils considered in the numerical study consist of two layers of identical thickness $H$ perfectly bonded together overlying a homogeneous halfspace with the material properties being given in Table 1. The influence of foundation shape is investigated first. Four types of rigid foundations resting on the multi-layered soils $(h=0)$ are considered in Figure 5, namely, rectangular, square, circular and cruciform, with the geometry and discretization being respectively shown in Figure 2(a) $(L=$ $2 H$ for rectangular and $L=H$ for square), Figure 2(b) and Figure 2(c). In Figure 5, the maximum amplitudes of nondimensional horizontal compliance (both real and imaginary parts) are observed in the cruciform foundation, followed by the circular, square and rectangular foundations respectively over the range of $0.1<\delta<3$. It is evident that a foundation with higher contact area would experience lower horizontal displacement than the one with lower area under the same amplitude of horizontal load $F_{\mathrm{x}}$.
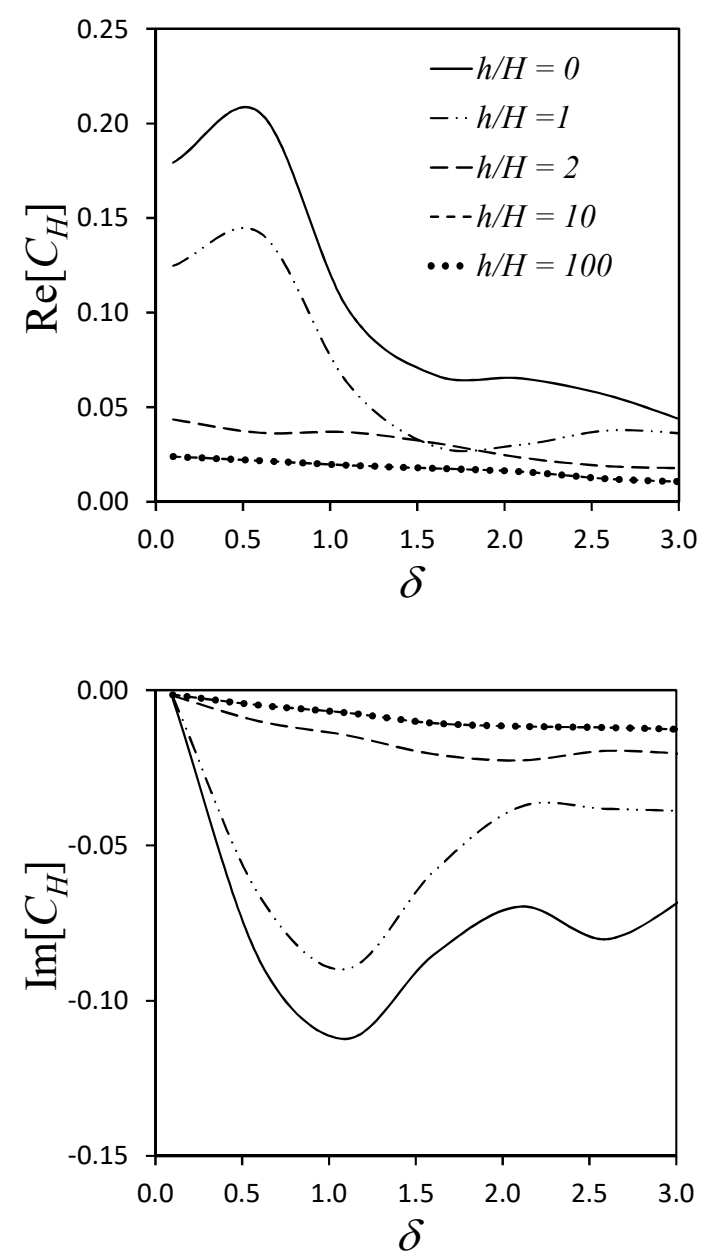

Figure 6. Non-dimensional horizontal compliances of rigid square foundations for different values of embedded depth.

The influence of the embedded depth " $h$ " on nondimensional horizontal compliances is presented in Figure 6 for the case of a rigid square foundation $(2 H$ $\times 2 H$ ). Five different values of embedded depths, i.e., $h / H=0,1,2,10$ and 100, are shown in Figure 6. It can be found that both real and imaginary parts of $C_{H}$ show oscillatory variation with the frequency for a small embedded depth when $h / H$ is less than two due to the effect of standing waves generated between the foundation and the layer interface that virtually disappear for very deeply buried foundation (a large value of $h / H$ ). It can be noticed that the horizontal compliances for the cases of $h / H=10$ and 100 are virtually identical indicating that the analysis of a deeply embedded foundation (i.e., $h / H>10$ ) in this multi-layered soil medium can be performed by considering the case of an embedded foundation in a poroelastic full-space and employing the material properties of the half-space given in Table 1 . 
Table 1. Material properties of a multi-layered poroelastic half-space considered in the numerical study

\begin{tabular}{lllllllll}
\hline & $\mu^{\dagger}$ & $\lambda^{\dagger}$ & $M^{\dagger}$ & $\rho^{\dagger \dagger}$ & $\rho_{f}^{\dagger \dagger}$ & $m^{\dagger \dagger}$ & $\alpha$ & $b^{\#}$ \\
\hline First layer & 2.5 & 5 & 25 & 2 & 1 & 3 & 0.95 & 1.5 \\
Second layer & 1.25 & 1.88 & 18.8 & 1.6 & 1 & 1.8 & 0.98 & 0.75 \\
Half-space & 10 & 10 & 20 & 2.4 & 1 & 4.8 & 0.9 & 4.5 \\
\hline
\end{tabular}

${ }^{\dagger} \times 10^{8} \mathrm{~N} / \mathrm{m}^{2} ;{ }^{\dagger+} \times 10^{3} \mathrm{~kg} / \mathrm{m}^{3} ;{ }^{\sharp} \times 10^{6} \mathrm{~N} \mathrm{~s} / \mathrm{m}^{4}$

Next, the influence of poroelastic material properties on non-dimensional horizontal compliances is investigated. It is found that the parameter $b$, which takes into account the internal friction between the solid matrix and the pore fluid, has a strong influence on the dynamic interaction between foundations and poroelastic media (Zeng and Rajapakse, 1999; Jin and Liu, 2000; Senjuntichai, Keawsawasvong and Plangmal, 2018a). To investigate the influence of the parameter $b$, Figure 7 presents non-dimensional horizontal compliances of the square foundation resting on three multi-layered systems with different values of the parameter $b$ of the first layer, i.e. $b^{(1)}=1.5 \times 10^{4} \mathrm{~N}$ $\mathrm{s} / \mathrm{m}^{4}, 1.5 \times 10^{6} \mathrm{~N} \mathrm{~s} / \mathrm{m}^{4}$ and $1.5 \times 10^{8} \mathrm{~N} \mathrm{~s} / \mathrm{m}^{4}$ respectively. It is noted that the first layer becomes less permeable with increasing values of $b^{(1)}$ since the parameter $b$ is inversely proportional to permeability. It can be seen from Figure 7 that the parameter $b$ has a significant influence on both real and imaginary parts of horizontal compliances, and the system is stiffer and less damped when the value of $b^{(1)}$ increases.

Figure 8 presents non-dimensional horizontal compliances of the square foundation resting on different supporting soil systems, i.e., a multi-layered system, a homogeneous half-space and a homogeneous layer of thickness $H$ with rigid base. The geometry and material properties of the multi-layered system are identical to that considered in Figures 5 and 6 whereas the material properties of homogeneous half-space and layer are identical to the first layer in Table 1. It is found from Figure 8 that dimensional horizontal compliance of the foundation on a homogeneous half-space varies smoothly over the frequency range $0.1<\delta<3$ for both real and imaginary parts whereas $C_{H}$ of the other two systems show oscillatory variations with the frequency due to the effect of from the standing waves generated in these two media.
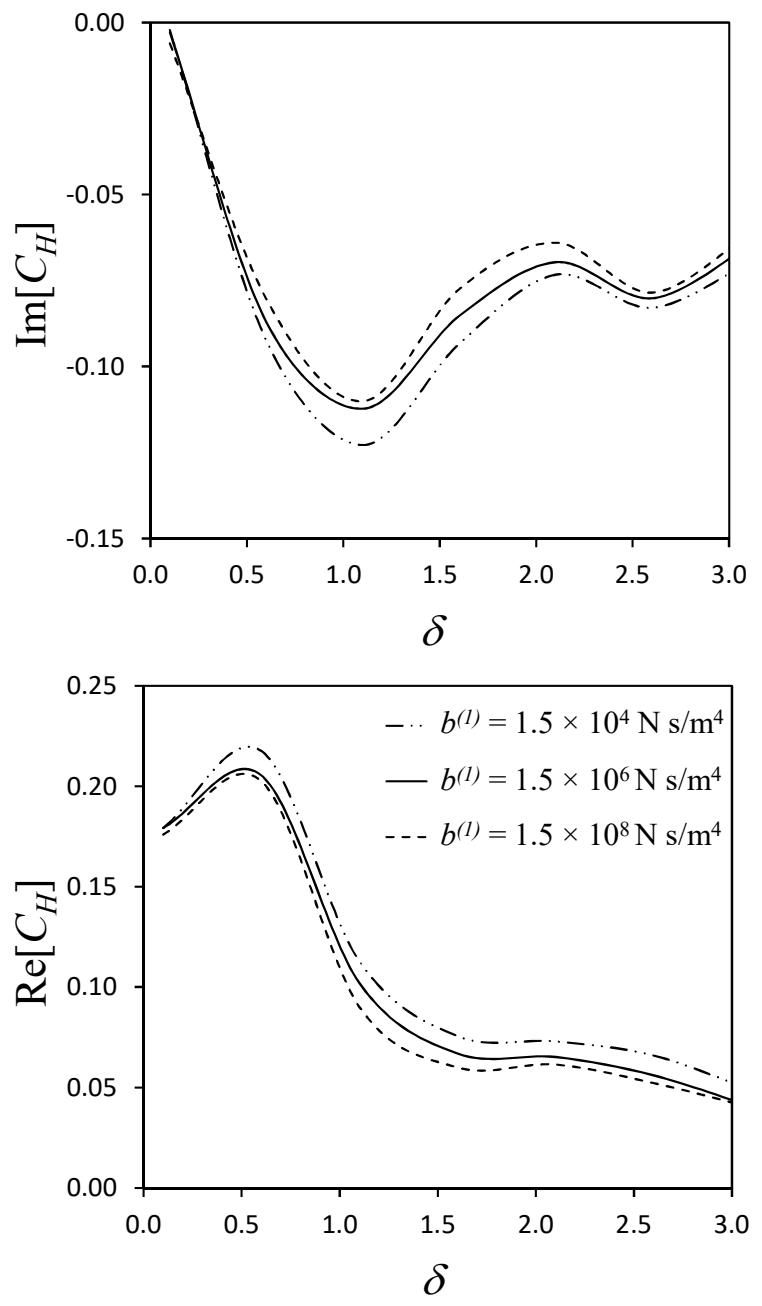

Figure 7. Non-dimensional horizontal compliances of rigid square foundation for different values of $b^{(1)}$. 

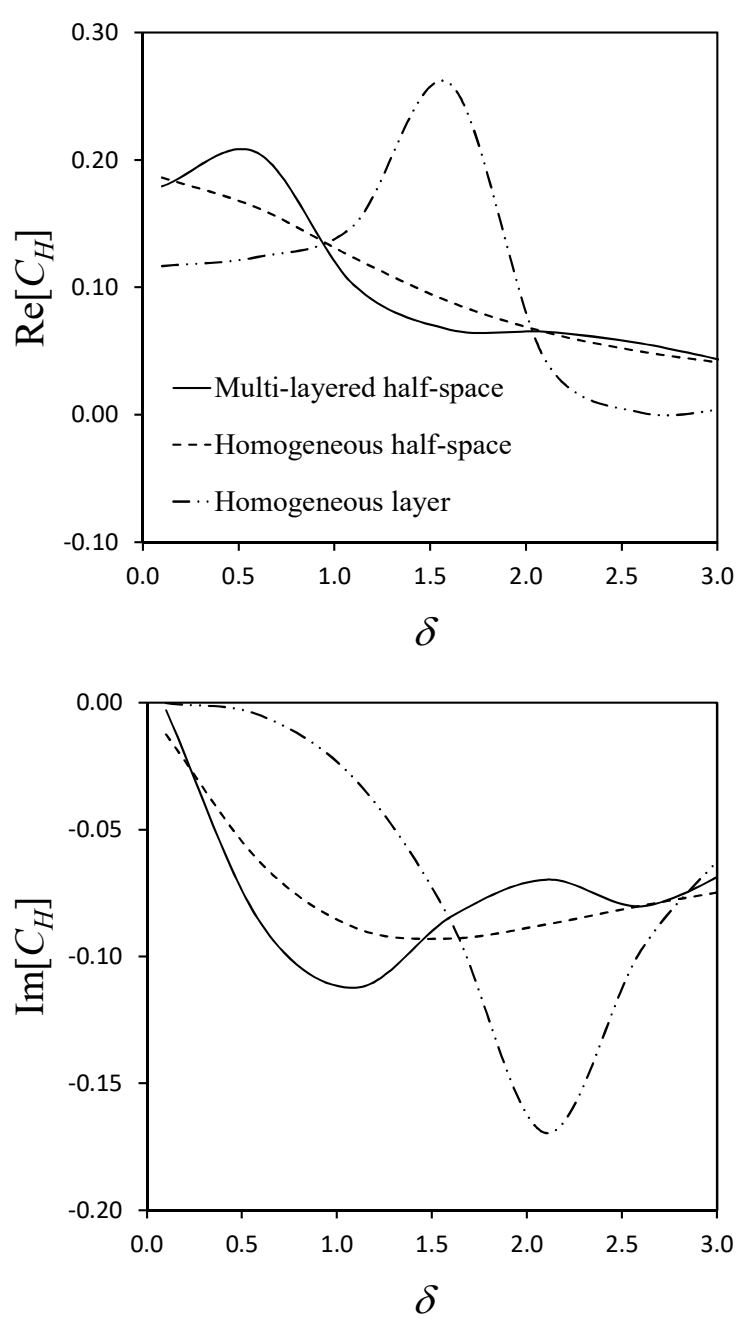

Figure 8. Non-dimensional horizontal compliances of rigid square foundations on different supporting soil systems.

\section{CONCLUSIONS}

The dynamic interaction between an embedded rigid foundation of arbitrary shape, subjected to timeharmonic horizontal loading, and multi-layered poroelastic soils are investigated in this paper by employing a discretization technique and an exact stiffness matrix scheme. The accuracy of the present solution is confirmed by comparing with an existing solution for compliances of rigid foundations bonded to homogeneous elastic and poroelastic half-spaces. Numerical results presented in this paper indicate that non-dimensional horizontal compliances of rigid foundations are significantly influenced by the shape of foundation, embedded depth, the parameter $b$, and the frequency of excitation. In addition, numerical results also show that the horizontal compliance of a rigid foundation on a layered medium is substantially different from the one on a homogeneous half-space due to the effect of the standing waves generated within the finite layer.

\section{ACKNOWLEDGMENTS}

The work was supported by the Thailand Research Fund [grant numbers PHD/0064/2558].

\section{REFERENCES}

Biot, M. A. (1941) 'General theory of three-dimensional consolidation', Journal of applied physics. AIP, 12(2), pp. 155-164.

Biot, M. A. (1956) 'Theory of propagation of elastic waves in a fluid-saturated porous solid. II. Higher frequency range', The Journal of the acoustical Society of america. ASA, 28(2), pp. 179-191.

Biot, M. A. (1962) 'Mechanics of deformation and acoustic propagation in porous media', Journal of applied physics. AIP, 33(4), pp. 1482-1498.

He, R. and Wang, L. (2013) 'Horizontal vibration of a rigid disk buried in a poroelastic half-space in contact with a fluid half-space', Soil Dynamics and Earthquake Engineering. Elsevier, 44, pp. 38-41.

He, R. and Zhou, C. (2015) 'Horizontal vibration of an underwater foundation on a poroelastic stratum', Procedia Engineering. Elsevier, 116, pp. 897-904.

Jin, B. and Liu, H. (2000) 'Horizontal Vibrations of a Disk on Poroelastic Half-Space', Soil Dynamics and Earthquake Engineering. doi: 10.1016/S02677261(00)00014-2.

Kassir, M. K. and Jimin, X. (1988) 'Interaction functions of a rigid strip bonded to saturated elastic half-space', International Journal of Solids and Structures. Elsevier, 24(9), pp. 915-936.

Lu, J.-F. and Jeng, D.-S. (2007) 'A half-space saturated poro-elastic medium subjected to a moving point load', International Journal of Solids and Structures. Elsevier, 44(2), pp. 573-586.

Luco, J. E. and Westmann, R. A. (1972) 'Dynamic response of a rigid footing bonded to an elastic half space', Journal of Applied Mechanics. American Society of Mechanical Engineers, 39(2), pp. 527-534.

Piessens, R. et al. (2012) Quadpack: a subroutine package for automatic integration. Springer Science \& Business Media.

Senjuntichai, T., Keawsawasvong, S. and Plangmal, R. (2018a) 'Three-dimensional dynamic response of multilayered poroelastic media', Marine Georesources \& Geotechnology. Taylor \& Francis, pp. 1-14. 
Senjuntichai, T., Keawsawasvong, S. and Plangmal, R. (2018b) 'Vertical vibrations of rigid foundations of arbitrary shape in a multi-layered poroelastic medium', Computers and Geotechnics. Elsevier, 100, pp. 121-134.

Sneddon, I. N. (1951) 'Fourier Transforms McGraw-Hill Book Co’, Inc., New York.

Urlich, C. M. and Kuhlemeyer, R. L. (1973) 'Coupled rocking and lateral vibrations of embedded footings', Canadian Geotechnical Journal. NRC Research Press, 10(2), pp. 145-160.
Wong, H. L. and Luco, J. E. (1976) 'Dynamic response of rigid foundations of arbitrary shape', Earthquake Engineering \& Structural Dynamics. Wiley Online Library, 4(6), pp. 579-587.

Wong, H. L. and Luco, J. E. (1978) 'Dynamic response of rectangular foundations to obliquely incident seismic waves', Earthquake Engineering \& Structural Dynamics. Wiley Online Library, 6(1), pp. 3-16.

Zeng, X. and Rajapakse, R. (1999) 'Vertical vibrations of a rigid disk embedded in a poroelastic medium', International Journal for Numerical and Analytical Methods in Geomechanics. Wiley Online Library, 23(15), pp. 2075-2095. 